Barker, P. F., Kennett, J. P., et al., 1990

Proceedings of the Ocean Drilling Program, Scientific Results, Vol. 113

\title{
56. CARBON ISOTOPE STRATIGRAPHY OF BULK SEDIMENTS, ODP SITES 689 AND 690, MAUD RISE, ANTARCTICA ${ }^{1}$
}

\author{
N. J. Shackleton ${ }^{2}$ and M. A. Hall ${ }^{2}$
}

\begin{abstract}
Carbon isotope measurements were made on bulk sediments from the well preserved calcareous sequences recovered at ODP Sites 689 and 690 on the Maud Rise, Weddell Sea, Antarctica. The very positive $\delta^{13} \mathrm{C}$ values that characterize the late Paleocene and the rapid trend toward lighter values in the early Eocene established in other sites are clearly recorded here and may be of value for long-distance stratigraphic correlation. However, values in the late Eocene are significantly more positive than have been reported from other areas. The general pattern of the records from Sites 689 and 690 is sufficiently unlike those previously reported from lower latitudes that we suggest that carbon isotope data should be used only with considerable caution for correlating sequences from such high latitudes with lower latitude records.
\end{abstract}

\section{INTRODUCTION}

ODP Sites $689\left(64^{\circ} 31^{\prime} \mathrm{S}, 03^{\circ} 6^{\prime} \mathrm{E} ; 2080 \mathrm{~m}\right)$ and $690\left(65^{\circ} 10^{\prime} \mathrm{S}\right.$, $\left.1^{\circ} 12^{\prime} \mathrm{E} ; 2914 \mathrm{~m}\right)$ provided a unique opportunity to investigate carbonate ooze sequences under present day polar waters. Well preserved carbonates were recovered spanning most of the $\mathrm{Pa}$ leogene.

It is generally agreed that for most purposes stable isotope analyses of bulk sediment are of little value, and that in order to obtain useful paleoenvironmental information it is necessary to analyze controlled sizes of selected foraminiferal species, for example (Berger et al., 1978). However, it is necessary to analyze bulk sediment to obtain appropriate data for contributing to our understanding of the history of the ocean carbon budget. This contribution provides a high latitude data set that supplements the information obtained from Cenozoic sequences from midlatitudes (Shackleton and Hall, 1984; Renard et al., 1983) and low latitudes (Shackleton, Hall, and Bleil, 1985).

Although there are significant spatial variations in $\delta^{13} \mathrm{C}$ in ocean surface waters today (Kroopnick et al., 1977) spatial variability in the $\delta^{13} \mathrm{C}$ content of surface sediments is quite small compared with the range observed through the Cenozoic (Shackleton, 1987) which leads to the possibility that the bulk sediment record of $\delta^{13} \mathrm{C}$ may provide additional data for stratigraphic purposes. The second objective of this contribution is to provide the data for testing this concept.

\section{ANALYTICAL METHODS AND DATA}

Samples from Holes 689B, 690B, and 690C averaging a few $\mathrm{mg}$ in weight were dried and then vacuum roasted to remove organic contaminants. The sediment was reacted with $100 \%$ orthophosphoric acid at $90^{\circ} \mathrm{C}$ in a common acid bath and the evolved carbon dioxide analyzed in a VG SIRA 9 mass spectrometer. Analytical accuracy is better than $\pm 0.1 \%$ although overall reproducibility as judged by repeat analyses is sometimes not as good due to the heterogeneity of the sediment.

Measurements are listed in Tables 1, 2, and 3. As an aid to preliminary evaluation of the data, each sample is assigned an age based on magnetobiostratigraphy as used in Stott et al. (this volume).

\footnotetext{
${ }^{1}$ Barker, P. F., Kennett, J. P., et al., 1990. Proc. ODP, Sci. Results, 113: College Station, TX (Ocean Drilling Program).

2 University of Cambridge, Subdepartment of Quaternary Research, Godwin Laboratory, Free School Lane, Cambridge CB2 3RS, UK.
}

\section{DISCUSSION}

Figure 1 shows the $\delta^{13} \mathrm{C}$ record for both sites plotted on the same time scale as was used for the discussion of planktonic foraminiferal paleotemperature data by Stott et al. (this volume).

Our first observation is that at the level of resolution shown here there are no apparent differences between the two sites. This suggests that it might be fruitful to investigate the possibility of using high resolution $\delta^{13} \mathrm{C}$ stratigraphy to correlate the two sites with higher precision than can be achieved using conventional biostratigraphical methods. Although more accurate correlation could certainly also be achieved by quantitative biostratigraphical methods, $\delta^{13} \mathrm{C}$ analysis of bulk sediment is more rapid and requires little expertise.

Comparison with other records is revealing. The late Paleocene $\delta^{13} \mathrm{C}$ peak at around $60 \mathrm{Ma}$ and the very rapid decline at the Paleocene-Eocene boundary is very characteristic of the record as previously observed in other areas, and it seems likely that $\delta^{13} \mathrm{C}$ events in this time interval will be valuable for long distance correlation. However, the pattern of variation in the later Eocene and the Oligocene is somewhat different in character from the lower latitude sequences as previously summarized (Shackleton, 1986, fig. 3), although it does quite closely resemble that obtained from planktonic foraminifers in DSDP Leg 29 sediments (Shackleton and Kennett, 1975, fig. 4). Neither the well-marked peak in $\delta^{13} \mathrm{C}$ values at about $35 \mathrm{Ma}$, nor the minimum at around $30 \mathrm{Ma}$, are recorded in the record shown by Shackleton (1986, fig. 3). It may be that $\delta^{13} \mathrm{C}$ stratigraphy will be of particular value within the Antarctic-Subantarctic water masses but that caution should be used in applying the approach globally.

\section{ACKNOWLEDGMENTS}

We are very grateful to the shipboard party of ODP Leg 113 for the time they devoted to sampling for this project. We thank Julie Cartledge for help in the laboratory. Jim Kennett made comments on a draft of this MS that were in every case helpful. This work was carried out with the support of NERC grant GR3/3606.

\section{REFERENCES}

Berger, W. H., Killingley, J. S., and Vincent, E., 1978. Stable isotopes in deep-sea carbonates: Box Core ERDC-92, West Equatorial Pacific. Oceanologica Acta 1:203-216.

Kroopnick, P. M., Margolis, S. V., and Wong, C. S., 1977. $\delta^{13} \mathrm{C}$ variations in marine carbonate sediments as indicators of the $\mathrm{CO}_{2}$ balance between the atmosphere and oceans. In Andersen, N. R., and 
Malahoff, A. (Ed.), The Fate of fossil Fuel $\mathrm{CO}_{2}$ in the Oceans, New York (Plenum), 295-321.

Renard, M., Richebois, G., and Letolle, R., 1983. Trace element and stable isotope geochemistry of Paleocene to Coniacian carbonate samples from Hole 516F, Comparison with North Atlantic and Tethys sites. In Barker, P. F., Carlson, R. L., Johnson, D. A., et al., Init. Repts. DSDP, 72: Washington (U.S. Govt. Printing Office), 399420.

Shackleton, N. J., 1986. Paleogene stable isotope events. Palaeogeogr., Palaeoclimatol., Palaeoecol. 57:91-102.

Shackleton, N. J., 1987. The carbon isotope history of the Cenozoic. In Brooks, J., and Fleet, A. J. (Eds), Petroleum Source Rocks Oxford (Blackwell), 427-438.

Shackleton, N. J., and Hall, M. A., 1984. Carbon isotope data from Leg 74 sediments. In Moore, T. C., Rabinowitz, P. D., et al., Init. Repts. DSDP, 74: Washington (U.S. Govt. Printing Office), 613619.

Shackleton, N. J., Hall, M. A., and Bleil, U., 1985. Carbon isotope stratigraphy, Site 577. In Heath, G. R., Burckle, L. D., et al., Init. Repts. DSDP, 86: Washington (U.S. Govt. Printing Office), 503511.

Shackleton, N. J., and Kennett, J. P., 1975. Paleotemperature history of the Cenozoic and the initiation of Antarctic glaciation: oxygen and carbon isotope analyses in DSDP sites 277,279 , and 281 . In Kennett, J. P., Houtz, R. E., et al., Init. Repts. DSDP, 29: Washington (U.S. Govt. Printing Office), 743-755.

Date of initial receipt: 30 June 1989

Date of acceptance: 21 July 1989

Ms 113B-211

Table 1. Stable isotope analyses in ODP Hole 689B. Ages estimates as in Stott et al. (this volume).

\begin{tabular}{|c|c|c|c|c|c|}
\hline $\begin{array}{c}\text { Lab } \\
\text { reference }\end{array}$ & $\begin{array}{l}\text { Depth } \\
\text { (mbsf) }\end{array}$ & $\begin{array}{l}\text { Age } \\
(\mathrm{Ma})\end{array}$ & Sample & $\delta^{18} \mathrm{O}$ & $\delta^{13} \mathrm{C}$ \\
\hline S $87 / 791$ & 23.09 & 9.622 & bulk & 3.57 & 1.45 \\
\hline S 87/786 & 30.59 & 12.747 & bulk & 4.10 & 2.26 \\
\hline S 87/785 & 34.56 & 14.401 & bulk & 4.17 & 2.02 \\
\hline S $87 / 784$ & 36.09 & 15.039 & bulk & 4.10 & 2.26 \\
\hline S $87 / 783$ & 37.57 & 15.656 & bulk & 3.98 & 2.54 \\
\hline S $87 / 782$ & 39.05 & 16.272 & bulk & 4.07 & 2.55 \\
\hline S $87 / 780$ & 40.57 & 16.906 & bulk & 4.18 & 2.52 \\
\hline S $87 / 779$ & 42.07 & 17.531 & bulk & 4.18 & 2.41 \\
\hline S 87/771 & 53.70 & 22.377 & bulk & 1.99 & 1.92 \\
\hline S $87 / 770$ & 55.70 & 23.211 & bulk & 2.08 & 2.34 \\
\hline S $87 / 768$ & 58.20 & 24.252 & bulk & 2.39 & 2.62 \\
\hline S $87 / 767$ & 59.70 & 24.877 & bulk & 2.50 & 2.07 \\
\hline S $87 / 766$ & 61.20 & 25.502 & bulk & 2.46 & 1.72 \\
\hline S $87 / 765$ & 62.08 & 25.869 & bulk & 2.28 & 1.51 \\
\hline S 87/764 & 63.27 & 26.239 & bulk & 2.81 & 2.05 \\
\hline S $87 / 763$ & 64.77 & 26.416 & bulk & 3.13 & 2.60 \\
\hline S $87 / 762$ & 66.27 & 26.593 & bulk & 2.29 & 1.54 \\
\hline S 87/761 & 67.77 & 26.770 & bulk & 2.55 & 1.08 \\
\hline S $87 / 760$ & 69.27 & 26.947 & bulk & 2.48 & 1.46 \\
\hline S 87/759 & 70.77 & 27.123 & bulk & 2.46 & 1.51 \\
\hline S 87/758 & 72.87 & 27.371 & bulk & 2.33 & 1.81 \\
\hline S $87 / 757$ & 74.37 & 27.548 & bulk & 2.45 & 2.06 \\
\hline S $87 / 756$ & 75.87 & 27.724 & bulk & 2.40 & 1.69 \\
\hline S $87 / 755$ & 77.37 & 27.901 & bulk & 2.50 & 1.57 \\
\hline S $87 / 754$ & 78.87 & 28.098 & bulk & 2.57 & 1.45 \\
\hline S $87 / 753$ & 80.37 & 28.341 & bulk & 2.63 & 1.39 \\
\hline S $87 / 752$ & 82.48 & 28.684 & bulk & 2.26 & 1.94 \\
\hline S $87 / 2453$ & 82.48 & 28.684 & bulk & 1.94 & 1.73 \\
\hline S 87/751 & 83.98 & 28.927 & bulk & 2.43 & 1.47 \\
\hline S $87 / 2454$ & 83.98 & 28.927 & bulk & 1.97 & 1.20 \\
\hline S $87 / 750$ & 85.48 & 29.170 & bulk & 2.59 & 1.76 \\
\hline S $87 / 2455$ & 85.48 & 29.170 & bulk & 2.20 & 1.61 \\
\hline S $87 / 749$ & 86.98 & 29.414 & bulk & 2.70 & 1.36 \\
\hline S $87 / 2456$ & 86.98 & 29.414 & bulk & 2.27 & 1.12 \\
\hline S $87 / 748$ & 88.48 & 29.657 & bulk & 2.68 & 1.52 \\
\hline S $87 / 2457$ & 88.48 & 29.657 & bulk & 2.40 & 1.43 \\
\hline S $87 / 747$ & 89.98 & 29.900 & bulk & 2.45 & 1.33 \\
\hline S $87 / 2458$ & 89.98 & 29.900 & bulk & 2.14 & 1.21 \\
\hline S $87 / 746$ & 90.90 & 30.050 & bulk & 2.50 & 1.57 \\
\hline S $87 / 2459$ & 90.90 & 30.050 & bulk & 2.11 & 1.06 \\
\hline S $87 / 745$ & 92.10 & 30.244 & bulk & 2.54 & 1.63 \\
\hline S $87 / 2460$ & 92.10 & 30.244 & bulk & 2.20 & 1.38 \\
\hline
\end{tabular}

Table 1 continued).

\begin{tabular}{|c|c|c|c|c|c|}
\hline $\begin{array}{c}\text { Lab } \\
\text { reference }\end{array}$ & $\begin{array}{l}\text { Depth } \\
\text { (mbsf) }\end{array}$ & $\begin{array}{l}\text { Age } \\
\text { (Ma) }\end{array}$ & Sample & $\delta^{18} \mathrm{O}$ & $\delta^{13} \mathrm{C}$ \\
\hline S 87/744 & 93.60 & 30.488 & bulk & 2.51 & 1.34 \\
\hline S $87 / 2461$ & 93.60 & 30.488 & bulk & 2.32 & 1.42 \\
\hline S $87 / 743$ & 95.10 & 30.731 & bulk & 2.59 & 1.22 \\
\hline S $87 / 2462$ & 95.10 & 30.731 & bulk & 2.28 & 1.23 \\
\hline S $87 / 742$ & 96.60 & 30.974 & bulk & 2.89 & 1.32 \\
\hline S $87 / 2463$ & 96.60 & 30.974 & bulk & 2.67 & 1.31 \\
\hline S $87 / 741$ & 98.10 & 31.218 & bulk & 2.59 & 1.49 \\
\hline S $87 / 2464$ & 98.10 & 31.218 & bulk & 2.28 & 1.45 \\
\hline S $87 / 740$ & 101.78 & 31.815 & bulk & 2.20 & 1.79 \\
\hline S $87 / 2249$ & 101.78 & 31.815 & bulk & 2.01 & 1.89 \\
\hline S $87 / 2465$ & 101.78 & 31.815 & bulk & 2.14 & 1.79 \\
\hline S 87/739 & 103.28 & 32.058 & bulk & 2.38 & 1.44 \\
\hline S $87 / 2250$ & 103.28 & 32.058 & bulk & 2.26 & 1.30 \\
\hline S $87 / 2466$ & 103.28 & 32.058 & bulk & 2.44 & 1.53 \\
\hline S 87/738 & 104.78 & 32.385 & bulk & 2.11 & 1.62 \\
\hline S $87 / 2467$ & 104.78 & 32.385 & bulk & 1.99 & 1.62 \\
\hline S 87/737 & 106.28 & 32.735 & bulk & 2.06 & 2.07 \\
\hline S $87 / 2468$ & 106.28 & 32.735 & bulk & 2.09 & 2.04 \\
\hline S 87/736 & 107.78 & 33.092 & bulk & 2.17 & 1.94 \\
\hline S $87 / 2469$ & 107.78 & 33.092 & bulk & 2.06 & 1.88 \\
\hline S $87 / 735$ & 109.28 & 33.450 & bulk & 2.17 & 1.72 \\
\hline S $87 / 2470$ & 109.28 & 33.450 & bulk & 1.99 & 1.61 \\
\hline S $87 / 734$ & 110.19 & 33.668 & bulk & 2.03 & 1.83 \\
\hline S $87 / 733$ & 111.39 & 33.955 & bulk & 2.30 & 1.93 \\
\hline S $87 / 2243$ & 111.39 & 33.955 & bulk & 2.01 & 1.90 \\
\hline S $87 / 732$ & 112.89 & 34.313 & bulk & 2.08 & 2.04 \\
\hline S $87 / 2244$ & 112.89 & 34.313 & bulk & 1.89 & 1.89 \\
\hline S 87/731 & 114.90 & 34.794 & bulk & 2.18 & 1.95 \\
\hline S $87 / 2245$ & 114.90 & 34.794 & bulk & 1.99 & 1.95 \\
\hline S $87 / 730$ & 116.40 & 35.152 & bulk & 2.37 & 2.19 \\
\hline S $87 / 729$ & 117.90 & 35.510 & bulk & 2.34 & 2.61 \\
\hline S $87 / 2247$ & 117.90 & 35.510 & bulk & 2.20 & 2.76 \\
\hline S $87 / 728$ & 119.92 & 35.993 & bulk & 2.17 & 2.43 \\
\hline S $87 / 2248$ & 119.92 & 35.993 & bulk & 2.19 & 2.63 \\
\hline S $87 / 727$ & 120.98 & 36.247 & bulk & 1.50 & 2.47 \\
\hline S $87 / 2236$ & 120.98 & 36.247 & bulk & 1.27 & 2.67 \\
\hline S $87 / 726$ & 122.48 & 36.605 & bulk & 1.31 & 2.39 \\
\hline S $87 / 2237$ & 122.48 & 36.605 & bulk & 1.09 & 2.48 \\
\hline S $87 / 725$ & 123.98 & 36.964 & bulk & 1.48 & 2.54 \\
\hline S $87 / 2238$ & 123.98 & 36.964 & bulk & 1.31 & 2.58 \\
\hline S $87 / 724$ & 125.48 & 37.343 & bulk & 1.71 & 2.50 \\
\hline S 87/2239 & 125.48 & 37.343 & bulk & 1.23 & 2.52 \\
\hline S $87 / 723$ & 126.98 & 37.706 & bulk & 1.31 & 2.84 \\
\hline S $87 / 2240$ & 126.98 & 37.706 & bulk & 1.33 & 3.07 \\
\hline S $87 / 722$ & 128.48 & 38.063 & bulk & 1.45 & 2.94 \\
\hline S $87 / 2241$ & 128.48 & 38.063 & bulk & 1.15 & 2.92 \\
\hline S $87 / 721$ & 129.38 & 38.277 & & 1.05 & 2.60 \\
\hline S $87 / 2242$ & 129.38 & 38.277 & bulk & 1.12 & 2.74 \\
\hline S $87 / 720$ & 130.72 & 38.574 & & 1.12 & $1.81^{* *}$ \\
\hline S $87 / 2229$ & 130.72 & 38.574 & bulk & 0.90 & 2.22 \\
\hline S $87 / 719$ & 132.22 & 38.856 & & 1.28 & $2.06^{* *}$ \\
\hline S $87 / 2230$ & 132.22 & 38.856 & bulk & 0.84 & 2.73 \\
\hline S $87 / 718$ & 133.72 & 39.109 & bulk & 1.40 & $2.06^{* *}$ \\
\hline S $87 / 2231$ & 133.72 & 39.109 & bulk & 0.97 & 2.03 \\
\hline S 87/717 & 135.22 & 39.362 & bulk & 1.12 & $1.86^{* *}$ \\
\hline S $87 / 2232$ & 135.22 & 39.362 & bulk & 1.23 & 1.93 \\
\hline S $87 / 716$ & 136.72 & 39.615 & bulk & 1.17 & $2.14^{* *}$ \\
\hline S $87 / 2233$ & 136.72 & 39.615 & bulk & 1.31 & 2.10 \\
\hline S $87 / 715$ & 138.22 & 39.868 & bulk & 1.08 & $1.99 * *$ \\
\hline S $87 / 2234$ & 138.22 & 39.868 & bulk & 1.28 & 1.90 \\
\hline S $87 / 714$ & 139.10 & 40.016 & bulk & 1.04 & $2.02^{* *}$ \\
\hline S $87 / 2235$ & 139.10 & 40.016 & bulk & 1.44 & 2.18 \\
\hline S $87 / 713$ & 139.22 & 40.037 & bulk & 0.99 & $1.96^{* *}$ \\
\hline S $87 / 2222$ & 139.22 & 40.037 & FINE & 1.45 & 2.27 \\
\hline S $87 / 712$ & 140.72 & 40.290 & bulk & 0.96 & $2.16^{* *}$ \\
\hline S $87 / 2223$ & 140.72 & 40.290 & $<64 \mu \mathrm{m}$ & 1.26 & 2.16 \\
\hline S 87/711 & 142.22 & 40.543 & bulk & 0.84 & $2.14^{* *}$ \\
\hline S $87 / 2224$ & 142.22 & 40.543 & $<64 \mu \mathrm{m}$ & 1.33 & 2.32 \\
\hline S $87 / 710$ & 143.72 & 40.795 & bulk & 0.77 & $2.26^{* *}$ \\
\hline S $87 / 2225$ & 143.72 & 40.795 & $<64 \mu \mathrm{m}$ & 0.87 & 1.82 \\
\hline S $87 / 709$ & 145.22 & 41.048 & bulk & 0.86 & $2.23^{* *}$ \\
\hline S $87 / 2226$ & 145.22 & 41.048 & $<64 \mu \mathrm{m}$ & 1.14 & 2.08 \\
\hline S $87 / 708$ & 146.72 & 41.301 & bulk & 0.67 & $2.26^{* *}$ \\
\hline S $87 / 2227$ & 146.72 & 41.301 & $<64 \mu \mathrm{m}$ & 0.98 & 2.07 \\
\hline S 87/707 & 147.58 & 41.446 & bulk & 0.15 & $2.22^{* *}$ \\
\hline S $87 / 809$ & 147.58 & 41.446 & bulk & 1.15 & 1.89 \\
\hline S $87 / 2228$ & 147.58 & 41.446 & $<64 \mu \mathrm{m}$ & 0.91 & 1.79 \\
\hline S 87/706 & 149.91 & 41.839 & bulk & 0.65 & $1.27 * *$ \\
\hline S $87 / 808$ & 149.91 & 41.839 & bulk & 1.18 & 2.07 \\
\hline
\end{tabular}


Table 1 continued).

\begin{tabular}{|c|c|c|c|c|c|}
\hline $\begin{array}{c}\text { Lab } \\
\text { reference }\end{array}$ & $\begin{array}{l}\text { Depth } \\
\text { (mbsf) }\end{array}$ & $\begin{array}{l}\text { Age } \\
(\mathrm{Ma})\end{array}$ & Sample & $\delta^{18} \mathrm{O}$ & $\delta^{13} \mathrm{C}$ \\
\hline $87 / 2441$ & 149.91 & 41.839 & $<64 \mu \mathrm{m}$ & 0.90 & 2.18 \\
\hline S 87/705 & 151.41 & 42.092 & bulk & 0.88 & $1.63 * *$ \\
\hline S $87 / 2442$ & 151.41 & 42.092 & $<64 \mu \mathrm{m}$ & 0.78 & 1.98 \\
\hline S $87 / 704$ & 152.91 & 42.345 & bulk & 0.51 & $1.88^{* *}$ \\
\hline S $87 / 806$ & 152.91 & 42.345 & bulk & 1.10 & 2.07 \\
\hline S $87 / 2443$ & 152.91 & 42.345 & $<64 \mu \mathrm{m}$ & 0.81 & 2.06 \\
\hline S $87 / 703$ & 154.41 & 42.598 & bulk & 0.57 & $1.72 * *$ \\
\hline S $87 / 805$ & 154.41 & 42.598 & bulk & 1.10 & 1.97 \\
\hline S $87 / 2444$ & 154.41 & 42.598 & $<64 \mu \mathrm{m}$ & 0.75 & 1.99 \\
\hline S $87 / 702$ & 155.91 & 42.851 & bulk & 0.79 & $1.77^{* * *}$ \\
\hline S $87 / 804$ & 155.91 & 42. & bulk & 1.03 & 2.20 \\
\hline S $87 / 2445$ & 155.91 & 42.8 & $<64 \mu \mathrm{m}$ & 0.71 & 2.06 \\
\hline S $87 / 701$ & 157.41 & 43.104 & bulk & 0.68 & $1.62^{* * *}$ \\
\hline S $87 / 803$ & 157.41 & 43.104 & bulk & 0.94 & 2.16 \\
\hline S $87 / 2446$ & 157.41 & 43.104 & $<64 \mu \mathrm{m}$ & 0.62 & 2.13 \\
\hline S $87 / 700$ & 158.28 & 43.251 & bulk & 1.13 & $2.43^{* *}$ \\
\hline S $87 / 802$ & 158.28 & 43.251 & bulk & 0.82 & 2.27 \\
\hline S $87 / 2201$ & 158.28 & 43.251 & $<64 \mu \mathrm{m}$ & 0.41 & 2.23 \\
\hline S $87 / 2447$ & 158.28 & 43.251 & $<64 \mu \mathrm{m}$ & 0.54 & 2.30 \\
\hline S $87 / 699$ & 159.62 & 43.477 & bulk & 1.05 & $2.81^{* *}$ \\
\hline S $87 / 801$ & 159.62 & 43.477 & bulk & 1.05 & 2.22 \\
\hline S $87 / 2202$ & 159.62 & 43.477 & $<64 \mu \mathrm{m}$ & 0.88 & 2.22 \\
\hline S $87 / 2448$ & 159 & 43 & $<64 \mu \mathrm{m}$ & 0.74 & 2.22 \\
\hline S $87 / 698$ & 161.12 & 43 & bulk & 1.09 & $2.17^{*}$ \\
\hline S $87 / 2203$ & 161.12 & 43. & $<64 \mu \mathrm{m}$ & 0.58 & 2.33 \\
\hline S $87 / 2449$ & 161.12 & 43. & $<64 \mu \mathrm{m}$ & 0.41 & 2.34 \\
\hline S $87 / 697$ & 162.62 & 43.983 & bulk & 1.43 & $2.02^{* *}$ \\
\hline S $87 / 2204$ & 162.62 & 43. & $<64 \mu \mathrm{m}$ & -0.04 & 2.21 \\
\hline S $87 / 2221$ & 162.62 & 43. & $<64 \mu \mathrm{m}$ & 0.05 & 2.26 \\
\hline S $87 / 2450$ & 162.62 & 43.983 & $<64 \mu \mathrm{m}$ & -0.07 & 2.20 \\
\hline S $87 / 695$ & 164.12 & 44.404 & bulk & 1.33 & $1.89^{* *}$ \\
\hline S $87 / 2205$ & 164.12 & 44.404 & $<64 \mu \mathrm{m}$ & 0.56 & 1.71 \\
\hline S $87 / 2451$ & 164.12 & 44.404 & $<64 \mu \mathrm{m}$ & 0.58 & 1.72 \\
\hline S $87 / 696$ & 165 & 44.669 & bulk & 1.55 & $2.23^{\circ}$ \\
\hline S $87 / 2206$ & 165 & 44. & $4 \mu \mathrm{m}$ & 0.43 & 1.45 \\
\hline S $87 / 2452$ & 165.62 & 44.669 & $<64 \mu \mathrm{m}$ & 0.40 & 1.37 \\
\hline S 87/694 & 169.32 & 45.136 & bulk & 1.65 & $2.21^{* *}$ \\
\hline S $87 / 2207$ & 169.32 & 45.136 & $<64 \mu \mathrm{m}$ & 0.30 & 1.82 \\
\hline S $87 / 693$ & 170.78 & 45.311 & bulk & 1.76 & $2.30 * *$ \\
\hline S $87 / 2208$ & 170.78 & 45.311 & $<64 \mu$ & 0.39 & 1.61 \\
\hline S $87 / 692$ & 172.32 & 45.495 & bulk & 1.64 & $2.20^{*}$ \\
\hline S 87/3609 & 172.32 & 45.495 & $<64 \mu$ & 0.56 & 1.63 \\
\hline S $87 / 691$ & 173.82 & 45.675 & bulk & 1.65 & $2.32^{*}$ \\
\hline S $87 / 2210$ & 173.82 & 45.675 & $<64 \mu \mathrm{m}$ & 0.53 & 1.63 \\
\hline S $87 / 690$ & 175 & 45 & bulk & 0.65 & 1.57 \\
\hline S $87 / 2211$ & 175.32 & 45.855 & $<64 \mu \mathrm{m}$ & 0.55 & 1.59 \\
\hline S $87 / 689$ & 176.02 & 45.938 & bulk & 0.61 & 1.50 \\
\hline S $87 / 2212$ & 176.82 & 46.034 & $<64 \mu \mathrm{m}$ & 0.52 & 1.54 \\
\hline S $87 / 688$ & 178.92 & 46.286 & bulk & 0.55 & 1.32 \\
\hline S $87 / 2213$ & 178.92 & 46.286 & $<64 \mu \mathrm{m}$ & 0.36 & 1.31 \\
\hline S $89 / 535$ & 179.26 & 46.326 & bulk & 0.44 & 1.42 \\
\hline S $87 / 687$ & 180.42 & 46.465 & bulk & 0.48 & 1.27 \\
\hline S $87 / 2214$ & 180.42 & 46.465 & $<64 \mu \mathrm{r}$ & 0.30 & 1.37 \\
\hline S 87/686 & 181.92 & 46.645 & bulk & 0.41 & 1.28 \\
\hline S $87 / 685$ & 183.42 & 46.824 & bulk & 0.44 & 1.42 \\
\hline S $87 / 2215$ & 183.42 & 46.824 & $<64 \mu \mathrm{m}$ & 0.25 & 1.41 \\
\hline S $87 / 684$ & 188.61 & 47.446 & bulk & 0.37 & 1.10 \\
\hline S $87 / 2216$ & 188.61 & 47.446 & $<64 \mu \mathrm{m}$ & 0.26 & 1.22 \\
\hline S $89 / 536$ & 190.46 & 47.667 & bulk & 0.17 & 1.09 \\
\hline S $87 / 683$ & 191.61 & 47.805 & bulk & 0.43 & 1.33 \\
\hline S $87 / 2217$ & 191.61 & 47.805 & $<64 \mu \mathrm{r}$ & 0.13 & 1.34 \\
\hline S $87 / 682$ & 198.31 & 48.607 & bulk & 0.15 & 1.67 \\
\hline S $87 / 2218$ & 198.31 & 48.607 & $<64$ & -0.01 & 1.66 \\
\hline S $87 / 681$ & 199.78 & 48.7 & bulk & 0.02 & 1.69 \\
\hline S $87 / 2219$ & 199.78 & 48. & $<64$ & -0 & 1.73 \\
\hline S $89 / 537$ & 200.16 & 48.828 & bulk & -0.07 & 1.65 \\
\hline S $87 / 680$ & 201.28 & 48.963 & bulk & 0.06 & 1.58 \\
\hline S $87 / 679$ & 202.78 & 49.142 & bulk & 0.22 & 1.70 \\
\hline S $87 / 678$ & 204.28 & 49.322 & bulk & 0.23 & 1.68 \\
\hline S $87 / 677$ & 205.10 & 50.753 & bulk & 0.11 & 1.65 \\
\hline S $87 / 676$ & 208.02 & 57.708 & bulk & -0.26 & 1.45 \\
\hline S $87 / 675$ & 209.52 & 58.118 & bulk & 0.08 & 2.18 \\
\hline S $89 / 538$ & 209.86 & 58.211 & bulk & -0.05 & 1.97 \\
\hline S $87 / 674$ & 211.02 & 58.528 & bulk & 0.22 & 2.61 \\
\hline S $87 / 673$ & 212.54 & 58.950 & bulk & 0.32 & 2.90 \\
\hline S $87 / 6$ & 237.09 & 67.275 & & 0.64 & 2.23 \\
\hline S $87 / 539$ & 237.27 & 67.313 & bulk & 0.43 & 1.95 \\
\hline
\end{tabular}

**Denotes analyses thought to be analytically unreliable.
Table 2. Isotopic analyses for ODP Hole 690B. Ages estimates as in Stott et al. (this volume).

\begin{tabular}{|c|c|c|c|c|c|}
\hline $\begin{array}{c}\text { Lab } \\
\text { reference }\end{array}$ & $\begin{array}{l}\text { Depth } \\
\text { (mbsf) }\end{array}$ & $\begin{array}{l}\text { Age } \\
(\mathrm{Ma})\end{array}$ & Sample & $\delta^{18} \mathrm{O}$ & $\delta^{13} \mathrm{C}$ \\
\hline S $87 / 668$ & 41.58 & 25.129 & bulk & 1.49 & 1.84 \\
\hline S 87/666 & 44.58 & 25.590 & bulk & 2.31 & 2.23 \\
\hline S $87 / 665$ & 46.08 & 25.821 & bulk & 2.66 & 2.04 \\
\hline S $87 / 664$ & 47.58 & 26.052 & bulk & 2.76 & 2.17 \\
\hline S $87 / 663$ & 49.08 & 26.283 & bulk & 2.75 & 2.17 \\
\hline S $87 / 662$ & 49.98 & 26.421 & bulk & 1.77 & 1.82 \\
\hline S $87 / 661$ & 51.18 & 26.606 & bulk & 2.26 & 1.62 \\
\hline S $87 / 660$ & 52.68 & 26.837 & bulk & 2.29 & 1.64 \\
\hline S $87 / 659$ & 54.13 & 27.060 & bulk & 2.33 & 1.83 \\
\hline S $87 / 658$ & 55.68 & 27.299 & bulk & 1.91 & 2.09 \\
\hline S $87 / 657$ & 57.18 & 27.530 & bulk & 2.25 & 1.95 \\
\hline S 87/656 & 58.68 & 27.761 & bulk & 2.28 & 2.19 \\
\hline S $87 / 655$ & 59.58 & 27.899 & bulk & 2.18 & 1.89 \\
\hline S $87 / 654$ & 60.85 & 28.095 & bulk & 2.63 & 1.46 \\
\hline S $87 / 653$ & 62.35 & 28.325 & bulk & 2.68 & 1.74 \\
\hline S $87 / 652$ & 63.85 & 28.556 & bulk & 2.68 & 1.29 \\
\hline S $87 / 651$ & 65.35 & 28.787 & bulk & 2.34 & 2.08 \\
\hline S $87 / 650$ & 66.85 & 29.018 & bulk & 2.41 & 1.36 \\
\hline S $87 / 649$ & 68.35 & 29.249 & bulk & 2.15 & 1.47 \\
\hline S $87 / 648$ & 69.27 & 29.391 & bulk & 2.41 & 1.63 \\
\hline S $87 / 647$ & 70.58 & 29.592 & bulk & 2.59 & 0.97 \\
\hline S $87 / 646$ & 72.08 & 29.823 & bulk & 2.42 & 1.32 \\
\hline S $87 / 645$ & 73.58 & 30.054 & bulk & 2.59 & 1.35 \\
\hline S $87 / 644$ & 75.08 & 30.285 & bulk & 2.51 & 1.47 \\
\hline S $87 / 643$ & 76.58 & 30.516 & bulk & 2.47 & 1.14 \\
\hline S $87 / 642$ & 78.08 & 30.747 & bulk & 2.69 & 1.24 \\
\hline S $87 / 641$ & 78.98 & 30.885 & bulk & 2.51 & 1.55 \\
\hline S $87 / 640$ & 80.18 & 31.070 & bulk & 2.59 & 1.32 \\
\hline S $87 / 639$ & 81.07 & 31.207 & bulk & 2.44 & 1.66 \\
\hline S $87 / 638$ & 81.68 & 31.301 & bulk & 2.23 & 1.10 \\
\hline S $87 / 637$ & 82.57 & 31.646 & bulk & 2.37 & 1.18 \\
\hline S $87 / 636$ & 83.18 & 31.916 & bulk & 2.27 & 1.77 \\
\hline S $87 / 635$ & 84.17 & 32.320 & bulk & 2.01 & 1.80 \\
\hline S $87 / 634$ & 84.68 & 32.528 & bulk & 1.86 & 2.19 \\
\hline S $87 / 633$ & 85.57 & 32.891 & bulk & 2.30 & 1.58 \\
\hline S $87 / 632$ & 86.18 & 33.140 & bulk & 2.37 & 1.70 \\
\hline S $87 / 631$ & 87.07 & 33.504 & bulk & 2.14 & 1.85 \\
\hline S $87 / 630$ & 87.68 & 33.753 & bulk & 2.30 & 1.86 \\
\hline S $87 / 629$ & 88.57 & 34.116 & bulk & 2.12 & 2.00 \\
\hline S $87 / 628$ & 89.88 & 34.651 & bulk & 2.43 & 2.05 \\
\hline S $87 / 627$ & 91.38 & 35.263 & bulk & 2.20 & 2.81 \\
\hline S $89 / 540$ & 91.76 & 35.418 & bulk & 2.19 & 2.47 \\
\hline S $87 / 626$ & 92.88 & 37.191 & bulk & 2.12 & 2.36 \\
\hline S $87 / 625$ & 94.38 & 38.614 & bulk & 1.64 & 2.06 \\
\hline S $87 / 624$ & 95.88 & 39.013 & bulk & 1.58 & 1.72 \\
\hline S $87 / 623$ & 97.38 & 39.412 & bulk & 1.53 & 1.95 \\
\hline S $87 / 622$ & 98.28 & 39.651 & bulk & 1.43 & 1.82 \\
\hline S $87 / 621$ & 99.58 & 39.997 & bulk & 1.39 & 1.92 \\
\hline S $87 / 620$ & 101.08 & 40.396 & bulk & 1.36 & 2.10 \\
\hline S $89 / 541$ & 101.47 & 40.500 & bulk & 1.44 & 2.01 \\
\hline S $87 / 619$ & 102.58 & 40.795 & bulk & 1.28 & 2.12 \\
\hline S $87 / 618$ & 104.08 & 41.194 & bulk & 1.09 & 2.18 \\
\hline S $87 / 617$ & 105.58 & 41.593 & bulk & 0.99 & 2.24 \\
\hline S $87 / 616$ & 107.08 & 44.445 & bulk & 0.95 & 1.56 \\
\hline S $87 / 615$ & 107.98 & 44.767 & bulk & 0.99 & 1.61 \\
\hline S $87 / 614$ & 109.28 & 44.930 & bulk & 0.97 & 1.44 \\
\hline S $89 / 542$ & 111.13 & 45.160 & bulk & 0.55 & 1.14 \\
\hline S $87 / 613$ & 112.28 & 45.304 & bulk & 0.92 & 1.64 \\
\hline S $87 / 612$ & 113.78 & 45.491 & bulk & 0.74 & 1.74 \\
\hline S $87 / 611$ & 115.28 & 45.678 & bulk & 0.71 & 1.64 \\
\hline S $87 / 610$ & 116.78 & 45.865 & bulk & 0.61 & 1.70 \\
\hline S $87 / 609$ & 117.67 & 45.976 & bulk & 0.53 & 1.59 \\
\hline S $87 / 608$ & 119.28 & 49.214 & bulk & 0.46 & 1.47 \\
\hline S $87 / 607$ & 120.78 & 49.586 & bulk & 0.32 & 1.49 \\
\hline S $89 / 543$ & 121.16 & 49.681 & bulk & 0.32 & 1.41 \\
\hline S $87 / 606$ & 122.28 & 49.959 & bulk & 0.37 & 1.57 \\
\hline S $87 / 605$ & 123.78 & 50.331 & bulk & 0.34 & 1.68 \\
\hline S $87 / 604$ & 125.28 & 50.703 & bulk & 0.38 & 1.59 \\
\hline S $87 / 603$ & 126.78 & 51.075 & bulk & 0.27 & 1.68 \\
\hline S $87 / 602$ & 127.67 & 51.296 & bulk & 0.11 & 1.63 \\
\hline S $87 / 600$ & 128.79 & 51.574 & bulk & 0.19 & 1.76 \\
\hline S $87 / 601$ & 128.89 & 51.599 & bulk & 0.21 & 1.75 \\
\hline S $87 / 599$ & 130.39 & 51.971 & bulk & 0.02 & 1.51 \\
\hline S $89 / 544$ & 130.76 & 52.063 & bulk & -0.08 & 1.43 \\
\hline S $87 / 597$ & 131.79 & 52.318 & bulk & -0.07 & $0.66^{*}$ \\
\hline S $87 / 598$ & 131.89 & 52.343 & bulk & 0.01 & 1.59 \\
\hline S 87/596 & 132.39 & 52.476 & bulk & 0.13 & $0.89^{*}$ \\
\hline S $87 / 595$ & 133.79 & 52.885 & bulk & 0.21 & 1.52 \\
\hline
\end{tabular}


Table 2 (continued).

\begin{tabular}{|c|c|c|c|c|c|}
\hline $\begin{array}{c}\text { Lab } \\
\text { reference }\end{array}$ & $\begin{array}{l}\text { Depth } \\
\text { (mbsf) }\end{array}$ & $\begin{array}{l}\text { Age } \\
(\mathrm{Ma})\end{array}$ & Sample & $\delta^{18} \mathrm{O}$ & $\delta^{13} \mathrm{C}$ \\
\hline S $87 / 594$ & 133.89 & 52.915 & bulk & 0.36 & 1.37 \\
\hline S $87 / 593$ & 135.39 & 53.354 & bulk & 0.05 & 1.47 \\
\hline S $87 / 592$ & 137.29 & 53.910 & bulk & 0.12 & 1.64 \\
\hline S 87/591 & 138.58 & 54.169 & bulk & -0.05 & 1.40 \\
\hline S $87 / 590$ & 140.08 & 54.451 & bulk & -0.08 & 0.92 \\
\hline S $89 / 545$ & 140.46 & 54.522 & bulk & 0.13 & 0.91 \\
\hline S $87 / 589$ & 141.58 & 54.732 & bulk & 0.12 & 1.15 \\
\hline S $87 / 588$ & 143.08 & 55.014 & bulk & 0.44 & 1.42 \\
\hline S $87 / 587$ & 144.58 & 55.296 & bulk & 0.13 & 1.34 \\
\hline S $87 / 586$ & 146.08 & 55.577 & bulk & 0.17 & 1.54 \\
\hline S $87 / 585$ & 148.30 & 55.995 & bulk & 0.10 & 1.54 \\
\hline S $87 / 921$ & 149.72 & 56.077 & bulk & 0.31 & 1.70 \\
\hline S $87 / 584$ & 149.80 & 56.082 & bulk & 0.22 & 1.54 \\
\hline S $87 / 922$ & 149.82 & 56.083 & bulk & 0.35 & 1.66 \\
\hline S $87 / 923$ & 149.92 & 56.089 & bulk & 0.41 & 1.77 \\
\hline S $87 / 924$ & 150.02 & 56.095 & bulk & 0.27 & 1.71 \\
\hline S $87 / 925$ & 150.15 & 56.102 & bulk & 0.20 & 1.73 \\
\hline S $89 / 546$ & 150.16 & 56.103 & bulk & 0.15 & 1.50 \\
\hline S 87/926 & 150.26 & 56.109 & bulk & 0.24 & 1.79 \\
\hline S $87 / 583$ & 151.30 & 56.169 & bulk & 0.11 & 1.47 \\
\hline S $87 / 927$ & 152.79 & 56.255 & bulk & -0.01 & 1.50 \\
\hline S $87 / 582$ & 152.80 & 56.255 & bulk & -0.05 & 1.34 \\
\hline S $87 / 928$ & 152.87 & 56.259 & bulk & 0.09 & 1.38 \\
\hline S $87 / 929$ & 152.94 & 56.264 & bulk & 0.15 & 1.36 \\
\hline S $87 / 930$ & 153.02 & 56.268 & bulk & 0.28 & 1.42 \\
\hline S $87 / 931$ & 153.09 & 56.272 & bulk & 0.28 & 1.51 \\
\hline S $87 / 932$ & 153.18 & 56.277 & bulk & 0.32 & 1.62 \\
\hline S $87 / 933$ & 153.28 & 56.283 & bulk & 0.35 & 1.56 \\
\hline S $87 / 934$ & 153.37 & 56.288 & bulk & 0.37 & 1.58 \\
\hline S $87 / 935$ & 153.44 & 56.292 & bulk & 0.36 & 1.58 \\
\hline S $87 / 936$ & 153.50 & 56.296 & bulk & 0.35 & 1.65 \\
\hline S $87 / 937$ & 153.60 & 56.302 & bulk & 0.23 & 1.69 \\
\hline S $87 / 938$ & 153.71 & 56.308 & bulk & 0.34 & 1.70 \\
\hline S $87 / 939$ & 153.82 & 56.314 & bulk & 0.33 & 1.68 \\
\hline S $87 / 940$ & 153.94 & 56.321 & bulk & 0.31 & 1.63 \\
\hline S $87 / 941$ & 154.04 & 56.327 & bulk & 0.43 & 1.67 \\
\hline S $87 / 942$ & 154.13 & 56.332 & bulk & 0.42 & 1.65 \\
\hline S $87 / 943$ & 154.22 & 56.337 & bulk & 0.38 & 1.59 \\
\hline S $87 / 581$ & 154.30 & 56.342 & bulk & -0.05 & 1.30 \\
\hline S $87 / 944$ & 154.34 & 56.344 & bulk & 0.36 & 1.59 \\
\hline S $87 / 945$ & 154.44 & 56.350 & bulk & 0.42 & 1.63 \\
\hline S $87 / 946$ & 154.55 & 56.357 & bulk & 0.43 & 1.64 \\
\hline S $87 / 947$ & 154.66 & 56.363 & bulk & 0.36 & 1.65 \\
\hline S $87 / 948$ & 154.76 & 56.369 & bulk & 0.39 & 1.66 \\
\hline S 87/991 & 154.76 & 56.369 & bulk & 0.04 & 1.66 \\
\hline S $87 / 949$ & 154.84 & 56.373 & bulk & 0.44 & 1.65 \\
\hline S 87/992 & 154.84 & 56.373 & bulk & 0.06 & 1.62 \\
\hline S $87 / 950$ & 154.95 & 56.380 & bulk & 0.37 & 1.64 \\
\hline S $87 / 993$ & 154.95 & 56.380 & bulk & 0.13 & 1.51 \\
\hline S 87/951 & 155.01 & 56.383 & bulk & 0.30 & 1.65 \\
\hline S $87 / 994$ & 155.01 & 56.383 & bulk & 0.02 & 1.57 \\
\hline S $87 / 952$ & 155.12 & 56.389 & bulk & 0.26 & 1.66 \\
\hline S $87 / 995$ & 155.12 & 56.389 & bulk & 0.05 & 1.62 \\
\hline S $87 / 953$ & 155.22 & 56.395 & bulk & 0.37 & 1.72 \\
\hline S $87 / 996$ & 155.22 & 56.395 & bulk & 0.15 & 1.67 \\
\hline S $87 / 954$ & 155.33 & 56.402 & bulk & 0.32 & 1.65 \\
\hline S 87/997 & 155.33 & 56.402 & bulk & 0.07 & 1.63 \\
\hline S $87 / 955$ & 155.42 & 56.407 & bulk & 0.19 & 1.56 \\
\hline S 87/998 & 155.42 & 56.407 & bulk & 0.13 & 1.64 \\
\hline S 87/956 & 155.52 & 56.413 & bulk & 0.00 & 1.42 \\
\hline S 87/999 & 155.52 & 56.413 & bulk & 0.04 & 1.53 \\
\hline S 87/957 & 155.62 & 56.418 & bulk & 0.21 & 1.59 \\
\hline S $87 / 1000$ & 155.62 & 56.418 & bulk & 0.21 & 1.63 \\
\hline S 87/958 & 155.71 & 56.424 & bulk & -0.15 & 1.10 \\
\hline S $87 / 1001$ & 155.71 & 56.424 & bulk & 0.17 & 1.56 \\
\hline S $87 / 580$ & 155.80 & 56.429 & bulk & 0.07 & 1.54 \\
\hline S 87/959 & 155.82 & 56.430 & bulk & 0.00 & 1.18 \\
\hline S $87 / 1002$ & 155.82 & 56.430 & bulk & 0.11 & 1.58 \\
\hline S $87 / 960$ & 155.92 & 56.436 & bulk & 0.07 & 1.28 \\
\hline S $87 / 1003$ & 155.92 & 56.436 & bulk & 0.11 & 1.62 \\
\hline S 87/961 & 156.02 & 56.441 & bulk & 0.13 & 1.39 \\
\hline S $87 / 1004$ & 156.02 & 56.441 & bulk & 0.15 & 1.59 \\
\hline S $87 / 962$ & 156.09 & 56.446 & bulk & 0.05 & 1.56 \\
\hline S $87 / 1005$ & 156.09 & 56.446 & bulk & 0.10 & 1.64 \\
\hline S $87 / 963$ & 156.21 & 56.452 & bulk & 0.10 & 1.58 \\
\hline S $87 / 1006$ & 156.21 & 56.452 & bulk & 0.09 & 1.66 \\
\hline S $87 / 964$ & 156.32 & 56.459 & bulk & 0.12 & 1.68 \\
\hline S $87 / 1007$ & 156.32 & 56.459 & bulk & 0.11 & 1.73 \\
\hline S $87 / 965$ & 156.42 & 56.456 & bulk & 0.11 & 1.59 \\
\hline
\end{tabular}

Table 2 (continued).

\begin{tabular}{|c|c|c|c|c|c|}
\hline $\begin{array}{c}\text { Lab } \\
\text { reference }\end{array}$ & $\begin{array}{l}\text { Depth } \\
\text { (mbsf) }\end{array}$ & $\begin{array}{l}\text { Age } \\
(\mathrm{Ma})\end{array}$ & Sample & $\delta^{18} \mathrm{O}$ & $\delta^{13} \mathrm{C}$ \\
\hline S $87 / 1008$ & 156.42 & 56.465 & bulk & 0.09 & 1.65 \\
\hline S 87/966 & 156.49 & 56.469 & bulk & 0.14 & 1.54 \\
\hline S $87 / 1009$ & 156.49 & 56.469 & bulk & 0.09 & 1.66 \\
\hline S $87 / 967$ & 156.58 & 56.474 & bulk & 0.12 & 1.59 \\
\hline S $87 / 1010$ & 156.58 & 56.474 & bulk & 0.12 & 1.60 \\
\hline S 87/579 & 156.67 & 56.479 & bulk & 0.02 & 1.47 \\
\hline S $87 / 968$ & 156.69 & 56.480 & bulk & 0.09 & 1.65 \\
\hline S $87 / 969$ & 156.80 & 56.487 & bulk & 0.14 & 1.58 \\
\hline S $87 / 970$ & 156.91 & 56.494 & bulk & 0.25 & 1.60 \\
\hline S 87/971 & 157.29 & 56.527 & bulk & 0.17 & 1.55 \\
\hline S 87/972 & 157.39 & 56.535 & bulk & 0.11 & 1.57 \\
\hline S $87 / 973$ & 157.50 & 56.545 & bulk & 0.21 & 1.62 \\
\hline S $87 / 974$ & 157.60 & 56.553 & bulk & 0.16 & 1.64 \\
\hline S $87 / 975$ & 157.71 & 56.561 & bulk & 0.19 & 1.63 \\
\hline S $87 / 976$ & 157.82 & 56.570 & bulk & 0.14 & 1.65 \\
\hline S $87 / 977$ & 157.93 & 56.578 & bulk & 0.18 & 1.63 \\
\hline S $87 / 578$ & 157.99 & 56.582 & bulk & 0.01 & 1.56 \\
\hline S $87 / 978$ & 158.03 & 56.585 & bulk & 0.15 & 1.61 \\
\hline S 87/979 & 158.13 & 56.593 & bulk & 0.08 & 1.59 \\
\hline S $87 / 980$ & 158.24 & 56.601 & bulk & 0.36 & 1.68 \\
\hline S $87 / 981$ & 158.36 & 56.610 & bulk & 0.10 & 1.60 \\
\hline S $87 / 982$ & 158.47 & 56.618 & bulk & 0.17 & 1.77 \\
\hline S $87 / 983$ & 158.59 & 56.628 & bulk & 0.14 & 1.83 \\
\hline S $87 / 577$ & 159.49 & 56.695 & bulk & -0.13 & 1.28 \\
\hline S $89 / 547$ & 159.86 & 56.723 & bulk & -0.01 & 1.31 \\
\hline S $87 / 576$ & I 60.99 & 56.808 & bulk & -0.11 & 1.47 \\
\hline S $87 / 575$ & 162.49 & 56.921 & bulk & -0.21 & 1.73 \\
\hline S $87 / 574$ & 163.99 & 57.034 & bulk & -0.03 & 1.66 \\
\hline S $87 / 573$ & 165.49 & 57.147 & bulk & -0.04 & 1.69 \\
\hline S $87 / 572$ & 166.36 & 57.212 & bulk & -0.37 & 1.50 \\
\hline S $87 / 571$ & 167.69 & 57.313 & bulk & -0.44 & 1.05 \\
\hline S $87 / 570$ & 169.19 & 57.426 & bulk & -0.46 & 0.50 \\
\hline S $89 / 548$ & 169.55 & 57.454 & bulk & -0.49 & 0.06 \\
\hline S $87 / 569$ & 170.69 & 57.540 & bulk & -0.18 & 1.37 \\
\hline S $87 / 568$ & 172.19 & 57.654 & bulk & 0.06 & 2.04 \\
\hline S $87 / 567$ & 173.69 & 57.767 & bulk & 0.07 & 2.69 \\
\hline S $87 / 566$ & 175.07 & 57.869 & bulk & 1.01 & $3.37^{* * *}$ \\
\hline S $87 / 565$ & 176.57 & 57.983 & bulk & 0.52 & 3.12 \\
\hline S $89 / 549$ & 176.95 & 58.012 & bulk & 0.33 & 2.71 \\
\hline S $87 / 564$ & 178.07 & 58.098 & bulk & 0.36 & 3.07 \\
\hline S $87 / 563$ & 179.57 & 58.212 & bulk & 0.22 & 2.96 \\
\hline S $87 / 562$ & 181.08 & 58.332 & bulk & 0.15 & 3.05 \\
\hline S $87 / 561$ & 182.58 & 58.445 & bulk & 0.21 & 3.10 \\
\hline S $87 / 560$ & 183.97 & 58.550 & bulk & 0.31 & 3.36 \\
\hline S $87 / 559$ & 185.98 & 58.686 & bulk & 0.25 & 3.51 \\
\hline S $87 / 558$ & 187.48 & 58.781 & bulk & 0.31 & 3.16 \\
\hline S $87 / 557$ & 188.98 & 58.875 & bulk & 0.42 & 3.59 \\
\hline S $87 / 556$ & 190.12 & 58.947 & bulk & 0.52 & 3.48 \\
\hline S $87 / 555$ & 190.48 & 58.970 & bulk & 0.29 & 3.42 \\
\hline S $87 / 554$ & 192.01 & 59.068 & bulk & 0.46 & 3.82 \\
\hline S $87 / 553$ & 193.51 & 59.163 & bulk & 0.61 & 3.86 \\
\hline S $87 / 552$ & 195.01 & 59.258 & bulk & 0.61 & 4.09 \\
\hline S 87/551 & 196.51 & 59.353 & bulk & 0.57 & 4.18 \\
\hline S $87 / 550$ & 198.79 & 59.504 & bulk & 0.68 & 4.26 \\
\hline S $87 / 549$ & 200.29 & 59.599 & bulk & 0.63 & 4.20 \\
\hline S $87 / 548$ & 201.79 & 59.693 & bulk & 0.49 & 4.21 \\
\hline S $87 / 547$ & 203.29 & 59.788 & bulk & 0.76 & 3.87 \\
\hline S $87 / 546$ & 204.97 & 59.896 & bulk & 0.56 & 3.49 \\
\hline S $87 / 545$ & 206.47 & 59.990 & bulk & 0.56 & 3.31 \\
\hline S $87 / 544$ & 207.97 & 60.085 & bulk & 0.76 & 3.25 \\
\hline S $87 / 543$ & 209.47 & 60.179 & bulk & 1.60 & $3.57^{* * *}$ \\
\hline S $87 / 542$ & 210.97 & 60.274 & bulk & 0.90 & 3.46 \\
\hline S $87 / 541$ & 212.47 & 60.368 & bulk & 0.72 & 3.12 \\
\hline
\end{tabular}

*Denotes samples with evidence of significant reworking. ***Denotes intensive dissolution. 
Table 3. Isotopic analyses for ODP Hole 690C. Ages estimates as in Stott et al. (this volume).

\begin{tabular}{lcccrc}
\hline $\begin{array}{c}\text { Lab } \\
\text { reference }\end{array}$ & $\begin{array}{c}\text { Depth } \\
\text { (mbsf) }\end{array}$ & $\begin{array}{c}\text { Age } \\
(\mathrm{Ma})\end{array}$ & Sample & $\delta^{18} \mathrm{O}$ & $\delta^{13} \mathrm{C}$ \\
\hline S $87 / 539$ & 224.31 & 62.087 & bulk & 0.56 & 2.21 \\
S $87 / 538$ & 225.83 & 62.311 & bulk & 0.41 & 2.17 \\
S $87 / 537$ & 227.36 & 62.537 & bulk & 0.48 & 2.23 \\
S $87 / 536$ & 228.80 & 62.750 & bulk & 0.69 & 2.27 \\
S $87 / 535$ & 230.35 & 62.979 & bulk & 0.69 & 2.49 \\
S $87 / 534$ & 231.85 & 63.200 & bulk & 0.59 & 2.67 \\
S $87 / 533$ & 233.98 & 63.633 & bulk & 0.41 & 2.21 \\
S 87/532 & 234.48 & 63.734 & bulk & 0.17 & 2.03 \\
S 87/531 & 235.98 & 64.039 & bulk & -0.16 & 2.37 \\
\hline
\end{tabular}

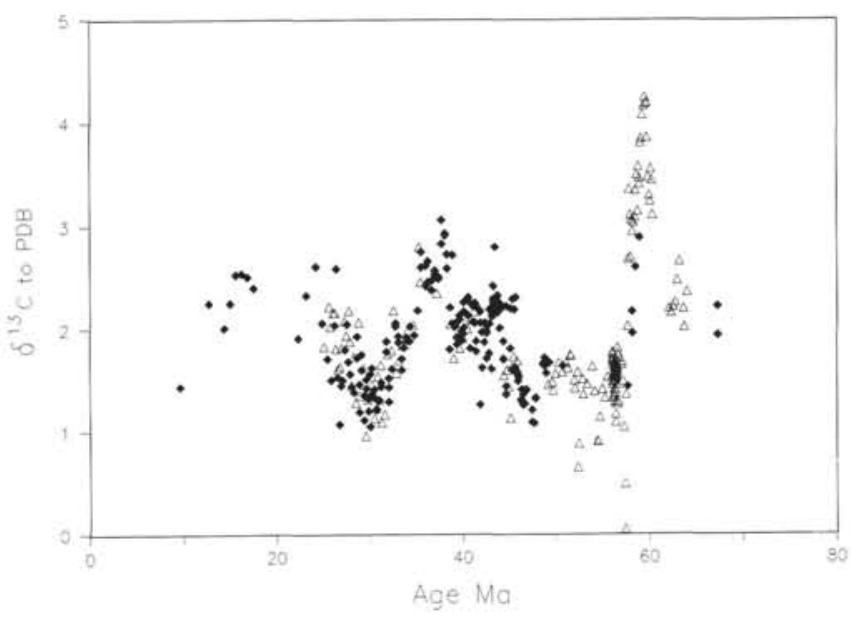

Figure $1 . \delta^{13} \mathrm{C}$ data for bulk sediment in ODP Sites 689 and 690 , using the time scale of Stott et al. (this volume). $\bullet=$ ODP Site $689 ; \Delta=$ ODP Site 690. 